\title{
ORIGINAL
}

\section{ESTADO DE SALUD DE LAS PERSONAS ANCIANAS Y HOSPITALIZACIÓN EN SERVICIOS GERIÁTRICOS, MÉDICOS Y QUIRÚRGICOS. ESTUDIO POBLACIONAL EN TOLEDO}

\author{
Francisco Suárez García (1), David Oterino de la Fuente (2), Salvador Peiró Moreno (2), \\ Francisco García García (1), Julián Librero (2), Alejandro Pérez Martín (1), Esperanza Martín \\ Correa (1) y Fernando Serrano Lira F (1)
}

(1) Hospital Virgen del Valle. Toledo

(2) Fundación Instituto de Investigación en Servicios de Salud

(*) Este trabajo ha sido financiado en parte por el Fondo de Investigación Sanitaria 94/1274

\section{RESUMEN}

Fundamento: El estado de salud y algunas características clínicas configuran un grupo de anciunos que necesitan más cuidados, que podrían beneficiarse de asistencia geriátrica especializada, aunque no existe consenso para identificar estos pacientes. El objetivo de este trabajo es describir el perfil de los pacientes mayores de 64 años ingresados en una unidad geriátrica y compararlo con los mayores de esta edad ingresados en los servicios médicos y quirúrgicos.

Método: Durante 18 meses se siguió una cohorte representativa de la población mayor de 64 años del Partido Judicial de Toledo $(n=3214)$, para identificar los ingresos y estancias hospitalarias en los hospitales públicos del área sanitaria. Las variables sobre estado de salud, se recogieron por entrevista personal, y los ingresos y sus características según datos del servicio de admisiones hospitalario.

Resultados: Ingresaron 410 personas $(12,8 \%)$, en geriatría 168 pacientes $(30.7 \%)$, en servicios médicos 204 $(37,3 \%)$ y en servicios quirúrgicos $174(32,0 \%)$. En geriatría la edad media fue significativamente mayor $(77,4$ años $)$, sin diferencias en la estancia media (12,8 días; IC95\%:10,6-14,0), fallecieron 44 pacientes $(8,1 \%)$, ingresados en geriatría 26 $(59,1 \%)$. En los servicios quirúrgicos ingresaron más mujeres, pacientes más jóvenes y con déficit leve de visión y audición; en geriatría, respecto a servicios médicos, más pacientes mayores de 80 años, viviendo en residencias, sin pareja, dependencia funcional moderada-severa, deterioro cognitivo, depresión, mala calidad de vida y escasos recursos sociales.

Conclusiones: No se observaron diferencias en el estado de salud entre los mayores de 64 años ingresados en servicios no quinúrgicos y quinírgicos. En geriatría, respecto a los otros grupos de servicios, los pacientes tuvieron una edad media más elevada, peor estado de salud, mayor mortalidad y estancia media similar.

Palabras clave: Estado de salud. Hospitalización. Ancianos. Geriatría.

Correspondencia:

David Oterino de la Fuente

El Puerto 46

33457 Sta. $\mathrm{M}^{2}$ del Mar. Asturias.Tel.: 985519989

Correo Electrónico: doterino@retemail.es

\section{ABSTRACT \\ Senior Citizen Health Conditions and Hospitilization on Geriatric, General and Surgical Floors. a Population Study Conducted in Toledo}

Background: The health condition and some clinical aspects configure a group of senior citizens in need of further care who could benefit from specialized geriatric care, although no consensus exists as to how to identify these patients. The aim of this study is to describe the profile of those patients over 64 years of age who are hospitalized in a geriatric unit and to compare this profile to the senior citizens hospitalized in general medical and surgical units.

Method: A cohort representative of the population over age 64 in the Judicial District of Toledo $(n=3214)$ was studied over an eighteen-month period for the purpose of identifying the income and length of stays at the public hospitals in the health care district in question. The health condition-related variables were gathered by means of personal interviews, and the income and the different aspects thereof by way of hospital admissions department data.

Results: A total of 410 individuals were admitted $(12.8 \%), 168$ patients $(30.7 \%)$ in geriatrics, $204(37.3 \%)$ in medical units and $174(32.0 \%)$ in surgical units. In geriatrics, the average age was significantly higher (age 77.4), there being no differences in the average length of stay (12.8 days; CI 95\%) 10.6-14.0), 44 patients $(8.1 \%$ ) having died, 26 $(59.1 \%)$ hospitalized in geriatrics. More females, younger patients having minor vision and hearing impairments were admitted to the surgical units. In geriatrics, as compared to the medical units, more patients over 80 years of age, living in senior citizen living facilities, having no spouse, moderate-tosevere functional dependence, impaired cognitive function, depression, poor quality of life and scanty social resources.

Conclusions: No differences were found to exist between the health conditions of those over age 64 who were hospitalized in non-surgical and surgical units. In geriatrics, as compared to the other groups of units, the patients were older, in worse condition, had a higher death rate and similar average length of stay.

Key words: Health Condition. Hospitalization. Senior Citizens. Geriatrics. 


\section{INTRODUCCIÓN}

El porcentaje de personas mayores de 65 años en España supera actualmente el $15 \%$ de la población y se estima que superará el $17 \%$ para el año 2005 , siendo el segmento de población con 80 o más años el que exhibe un mayor incremento relativo'. Esta situación de progresivo envejecimiento de la población -al margen de problemas sociales, políticos y económicos- conlleva una especial complejidad para los servicios sanitarios, por su asociación con el aumento del consumo de los mismos. Así, se ha señalado que la tasa de ingresos hospitalarios de las personas mayores de 65 años duplica la de la población general, y llega a triplicarse cuando se refiere al colectivo de personas de más de 80 años $^{2}$; igualmente, se ha observado una estancia media superior y un mayor numero de reingresos en las personas mayores $^{3,4}$. Esta situación estaría relacionada con los cambios demográficos, pero también con un incremento de la intensidad diagnóstica y terapéutica ${ }^{5,6}$.

El peor estado de salud y algunas características sociales (ancianos que viven solos), funcionales (limitaciones para realizar actividades de la vida cotidiana), clínicas (enfermedades crónicas, pluripatología) y mentales (deterioro cognitivo, depresión) son elementos característicos de un grupo particular de ancianos con necesidades específicas y una mayor utilización de los servicios. Esta problemática incluye mayores necesidades de enfermería, mayor presión sobre los cuidadores informales, mayor requerimiento de servicios sociales y, en conjunto, una mayor demanda de atención sanitaria y socio-sanitaria, tanto en el hospital como en el domicilio, así como con formulas mixtas (hospital de día y similares) $^{7-9}$.

Este grupo de pacientes geriátricos, que para algunos autores constituye el $10-25 \%$ de los ingresos en los servicios médicos de los hospitales de agudos ${ }^{4,10,11}$, serían candidatos a cuidados geriátricos especializados. Los beneficios de esta modalidad de aten- ción no están bien establecidos, existiendo literatura que refiere que los pacientes asistidos en unidades geriátricas especializadas tienen mejor estado funcional al alta, menor institucionalización posterior y menor mortalidad $^{12-14}$, así como costes similares respecto a otras alternativas ${ }^{15,16}$, mientras que otros trabajos no observan beneficios en el estado funcional tras el alta o los reingresos ${ }^{17}$. Estas diferencias podrían, al menos en parte, estar relacionadas con los distintos perfiles de personas atendidas en cada unidad geriátrica, ya que -aunque se han citado múltiples factores de riesgo y se han utilizado distintos instrumentos de valoración con esta finalidad- no existe un consenso sobre las características que definen a los pacientes susceptibles de beneficiarse del ingreso en geriatría frente al ingreso en otras unidades hospitalarias $^{4,11,18}$.

El objetivo de este trabajo es, precisamente, describir el perfil de la población mayor de 64 años ingresada en una unidad geriátrica y compararlo con el de las personas mayores de esta edad ingresadas en los servicios médicos y quirúrgicos, incluyendo en este perfil -además de las características clínicas y demográficas- aspectos relativos al estado funcional, capacidad cognitiva y calidad de vida, así como algunas características sociales y educativas de estas personas.

\section{MATERIAL Y MÉTODO}

Diseño: Cohorte prospectiva, integrada por una muestra representativa de la población mayor de 65 años del Partido Judicial de Toledo (PJT), que fue estudiada durante 18 meses (entre el 1/10/94 y el 31/3/96) para identificar sus ingresos y estancias hospitalarias en los hospitales públicos del área sanitaria.

Muestra: La muestra, proveniente de un estudio poblacional para estimar la prevalencia de demencia senil en personas mayores de 65 años (hospitalizados o no, pero ex- 
cluyendo los ingresados en hospitales psiquiátricos) del PJT, se obtuvo mediante un muestreo polietápico, estratificado según el índice de ruralidad de los municipios. El tamaño muestral $(n=3.937)$ se calculó para una prevalencia estimada de las demencias menos frecuentes del $0,75 \%$, con una precisión del $1 \%$ y un nivel de confianza del 95\%. A los individuos seleccionados se les envió una carta personalizada un mes antes de comenzar el estudio, en la que se les informaba de la naturaleza del mismo, su carácter voluntario y solicitándoles su colaboración para realizarles una entrevista en su domicilio; una semana después se concertó la cita telefónicamente. A 723 individuos no se les pudo realizar la entrevista, por lo que la muestra final fue de $n=3.214$ (tasa de respuesta: $81,6 \%$ ). Los 723 sujetos no entrevistados corresponden a 394 negativas, 129 desplazados, 50 fallecidos, 15 ingresados en el hospital, 54 no conocidos en el domicilio indicado y 81 debido a otras causas. En personas con déficit cognitivo o con dificultades en el lenguaje la información se obtuvo o se completó a través del cuidador principal. Las entrevistas se llevaron a cabo entre enero y septiembre de 1994 por psicólogos y médicos residentes del servicio de geriatría, previamente entrenados, mediante un cuestionario estructurado donde se recogía información sobre características personales, patología asociada, hábitos de vida y estado de salud.

Lugar del estudio: El PJT agrupa a 23 municipios (salvo el de Toledo, ninguno supera los 5.000 habitantes), con una población total de 101.900 habitantes, de los cuales $14.862(14,6 \%)$ tenían 65 o más años según el Censo de 1991. El PJT está incluido en el Area Sanitaria de Toledo, que cuenta con dos hospitales públicos: el Hospital Provincial (perteneciente a la Diputación Provincial) y el Complejo Hospitalario de Toledo (Insalud). El Complejo Hospitalario de Toledo está formado por un hospital general (Virgen de la Salud), un hospital monográfico de geriatría (Virgen del Valle) y el Hospital Nacional de Parapléjicos. Los servicios médicos (SM) y los servicios quirúrgicos $(\mathrm{SQ})$ se ubican en el hospital general, mientras que el servicio de geriatría (SG) se halla en el hospital monográfico y cuenta con 132 camas de agudos ${ }^{19}$.

El grupo de SM (155 camas) incluye los servicios de medicina interna, endocrinología, cardiología, digestivo, endocrinología, nefrología, neumología, neurología, oncología, reumatología y dermatología. El grupo de SQ (245 camas) los de cirugía general, cirugía plástica, traumatología, ginecología, urología, cirugía vascular, oftalmología, y ORL. Los servicios centrales son únicos, aunque el hospital geriátrico dispone de un laboratorio y servicio de radiología básico. El servicio de urgencias está ubicado en el hospital Virgen de la Salud. No existe un protocolo explícito que regule los criterios de ingreso en el servicio de geriatría, pero la pauta habitual es derivar a los pacientes que tienen entre 65 y 75 años con procesos médicos y pluripatología o patología incapacitante, y a todos los mayores de 75 años, tengan o no pluripatología, discapacidad u otras característica de anciano frágil.

Recogida de datos: Los datos sobre las características de los sujetos, estado de salud y patología autopercibida fueron recogidos por entrevista personal con el mismo y sus familiares; posteriormente se identificó, a través de las bases de datos de admisiones de los hospitales, a los sujetos que habían ingresado en alguna ocasión durante el periodo de estudio. No se incluyeron los ingresos producidos en el Hospital Nacional de Parapléjicos y en hospitales psiquiátricos. Los diagnósticos de alta fueron codificados según los 17 grupos de la Clasificación Internacional de Enfermedades $9^{\mathrm{a}}$ Revisión (CIE-9).

Variables: Se utilizaron las siguientes definiciones y variables:

Características personales: Edad, sexo, estado civil (casados, sin pareja), domicilio (propio, vive con familiares o cuida- 
dores, residencia), nivel de estudios (analfabetos, leer y escribir/sin estudios y estudios primarios, secundarios o superiores), recursos sociales (excelentes, buenos, incapacidad social).

Estado de Salud: autopercepción de salud valorada mediante la pregunta: $¿ D u$ rante la semana pasada, cómo se ha encontrado usted de salud? (muy bien, bien, mal o muy mal); caídas en el último año (ninguna, una o más); estado visual, (sin déficit, déficit leve: dificultad para leer o coser, déficit moderado-severo: dificultad para las actividades de la vida diaria); auditivo (sin déficit, déficit leve: dificultad para conversar en grupo o ambiente ruidoso; déficit moderado-severo: dificultad para conversar con una persona o imposibilidad de conversar); incontinencia esfínteres (si, no); estado cognitivo (Mini-Mental State Examination); estado funcional (Indice de Katz); depresión (Geriatric Depression Scale); calidad de vida (Quality Life Index); grado de movilidad, valorado según la forma en que podían caminar: sin ayudas, con uno o dos bastones, andador, ayudas de personas o imposibilidad para deambular (siempre que coexistían dos niveles funcionales se aceptaba el peor); se consideró movilidad plena si caminaban solos o con ayuda de un bastón y movilidad restringida el resto de las categorías.

Datos de la hospitalización: Número de ingresos, duración de la estancia, servicio de ingreso (medico, quirúrgico, geriatría), tipo de ingreso (urgente, programado), destino al alta (atención primaria, consultas externas, traslado de hospital y éxitus), diagnóstico principal al alta.

Instrumentos: Se utilizaron los siguientes instrumentos de medición:

Older Americans Resources and Services (OARS): Para evaluar la situación social general se empleó la traducción española del $\mathrm{OARS}^{20}$ en su área social, que ya ha demostrado su utilidad en nuestro país $^{21}$. Consta de una serie de preguntas que proporcionan información sobre estructura familiar, patrones de amistad, visitas sociales, disponibilidad de un confidente y disponibilidad de una persona que pueda prestar ayuda en casos de necesidad, se emplea una versión diferente para aquellas personas que viven en una residencia. Este instrumento mide la disponibilidad de recursos sociales en forma de la cantidad y calidad de las relaciones personales y de cuidados en los momentos de necesidad. A partir de las respuestas dadas por el sujeto, el evaluador hace un juicio de la situación social sobre una escala de 6 puntos, que va desde recursos excelentes hasta la total incapacidad social. En este trabajo se han considerado los siguientes grupos: recursos sociales excelentes, buenos e incapacidad social (incapacidad social leve, moderada, severa y total).

Mini-Mental State Examination: Es uno de los instrumentos más utilizados y con mayor rendimiento para la detección de deterioro cognitivo y demencia y ha demostrado una alta fiabilidad, validez, sensibilidad, especificidad y poder discriminativo ${ }^{22}$, incluyendo sus versiones en español ${ }^{23-25}$. Consta de 11 apartados que exploran la orientación, fijación, atención, cálculo, memoria y lenguaje siendo la puntuación máxima de 30 puntos. Es muy sensible para el nivel de estudios del individuo, por lo que puede dar falsos positivos en individuos con bajo nivel cultural o con déficits sensoria$\operatorname{les}^{26}$, por lo que se establecieron distintos puntos de corte según el nivel de estudios de los sujetos. Los puntos de corte para identificar el deterioro cognitivo, determinados en un estudio caso-control previo al trabajo de campo, fueron $17 / 18$ para los analfabetos, $20 / 21$ sin estudios y $23 / 24$ estudios primarios o superiores.

Indice de Katz: Es una de las escalas mejor estudiadas y más utilizadas para la evaluación de las actividades de la vida diaria $^{27}$. La medición requiere una evaluación dicotómica (dependencia/independencia) de seis funciones: bañarse, vestirse, ir al aseo, trasladarse, continencia de esfínteres y ali- 
mentación. Se da 1 punto por cada nivel de independencia. La puntuación final será la suma de las puntuaciones; por lo tanto el rango de la escala oscilará entre 6 (totalmente válido) y 0 (totalmente invalido). Se consideró independiente a aquel individuo que realiza la función y si no la realiza, aunque pudiera hacerlo, se considera dependiente. Independiente significa sin supervisión, dirección o ayuda activa, salvo las consideraciones particulares. Como en algún trabajo previo ${ }^{12}$ se consideraron independientes los individuos que realizaban todas las actividades (puntuación de 6) dependencia moderada si no realizaban 1 actividad (puntuación de 5), y dependencia severa si no realizaban 2 o más actividades (puntuación menor de 5).

Geriatric Depression Scale (GDS). La determinación de la presencia de depresión se realizó mediante la Geriatric Depression Scale (GDS) en su versión reducida ${ }^{28,29}$. Esta escala está formada por préguntas sencillas sobre el estado anímico, con contestación de sí/no. Según la respuesta a cada pregunta se puntúa 0-1, con máxima puntuación de 15 . Se consideró que existía depresión cuando la suma de los puntos obtenidos era superior a cinco $^{29}$.

Quality of Life Index. Es un índice inte grado30 que evalúa la calidad de vida del individuo, habiendo ya sido validado en España $^{31}$. Se le pregunta al sujeto, referido a la última semana, sobre su ocupación habitual, actividades de la vida diaria, salud, apoyo y estado de ánimo, puntuándose cada apartado con 2, 1 ó 0 . La puntuación máxima es de 10 y la mínima de 0 . En este trabajo se considero buena calidad de vida los individuos que puntuaron entre 7 y 10 mala calidad de vida los que puntuaron por debajo de 7 .

Análisis: Se describieron, en primer lugar, las características de la muestra global y, a continuación, se analizaron las características de los pacientes que tuvieron algún ingreso en los 18 meses siguientes a la fina- lización de la encuesta, en función del servicio de ingreso. Para valorar las diferencias en tales características entre servicios se utilizó la prueba de $\chi 2$ (con corrección de Yates o sustituida por la prueba exacta de Fisher cuando alguna categoría tuvo un recuento inferior a 5 ó 3 casos, respectivamente) $\mathrm{y}$, en el caso de las variables cuantitativas se empleó la prueba ANOVA de una vía. Todos los cálculos se realizaron con el programa SPSS®.

\section{RESULTADOS}

Características de la población: La tabla 1 muestra las características personales y el estado de salud de los 3.214 individuos de la muestra. La edad media fue de 74,1 años (rango 65-103), teniendo el 22,8\% de los individuos 80 o más años. Existía un predominio de las mujeres $(56,3 \%)$ en todos los grupos de edad, alcanzando $65,7 \%$, en el grupo de las personas mayores de 80 años. La mayor parte vivía en su propio domicilio $(81,2 \%)$, estando un $3,2 \%$ en residencias de ancianos. El $62,5 \%$ estaban casados y el $29,2 \%$ viudos. Respecto al nivel de estudios, predominaron los que solo sabían leer y escribir $(66,8 \%)$, con un porcentaje de analfabetismo del $19,1 \%$; el $85,8 \%$ de los sujetos tenían recursos sociales excelentes o buenos.

La mayoría de los sujetos estudiados eran independientes para realizar todas las actividades básicas de la vida diaria $(76,5 \%)$ y deambulaban solos o con ayuda de un bastón $(93,0 \%)$, referían tener un estado de salud bueno o muy bueno $(83,6 \%)$, sin problemas de depresión $(81,1 \%)$, buena calidad de vida $(85,9 \%)$, sin deterioro cognitivo $(72,9 \%)$, sin déficit de visión $(79,3 \%)$, ni audición $(82,8 \%)$, controlaban esfínteres $(83,4 \%)$, y no habían sufrido ninguna caída en el ultimo año $(82,3 \%)$. Las mujeres, las personas mayores de 80 años y las que vivían en residencias, presentaron una proporción significativamente mayor de analfa- 
Tabla 1

Características personales y estado de salud de la población

\begin{tabular}{|c|c|c|c|c|}
\hline & $n$ & $\%$ & Mujeres & $(\%)$ \\
\hline \multicolumn{5}{|l|}{ EDAD } \\
\hline $65-69$ & 966 & $(30.1)$ & 510 & $(52,8)$ \\
\hline $70-74$ & 892 & $(27,8)$ & 470 & $(52,0)$ \\
\hline $75-79$ & 616 & $(19,2)$ & 345 & $(56,0)$ \\
\hline 80 o más & 732 & $(22,8)$ & 481 & $(65,7)$ \\
\hline \multicolumn{5}{|l|}{ SEXO } \\
\hline Hombre & 1404 & 43,7 & & \\
\hline Mujer & 1810 & 56,3 & & \\
\hline \multicolumn{5}{|l|}{ ESTADO CIVIL } \\
\hline Casado & 1997 & $(62,5)$ & 897 & $(44,9)$ \\
\hline Sin Pareja & 1200 & $(37,5)$ & 898 & $(74,8)$ \\
\hline \multicolumn{5}{|l|}{ NIVEL DE ESTUDIOS } \\
\hline Analfabeto & 611 & $(19,1)$ & 451 & $(73,8)$ \\
\hline Leer/Escribir & 2133 & $(66,8)$ & 1138 & $(53,4)$ \\
\hline Primarios/Superiores & 450 & $(14,1)$ & 209 & $(46,4)$ \\
\hline \multicolumn{5}{|l|}{ DOMICILIO } \\
\hline Propia & 2568 & $(81,2)$ & 1375 & $(53,5)$ \\
\hline Cuidador & 497 & $(15,7)$ & 333 & $(67,0)$ \\
\hline Residencia & 98 & $(3,1)$ & 68 & $(69,4)$ \\
\hline \multicolumn{5}{|c|}{ RECURSOS SOCIALES (OARS) } \\
\hline Excelentes & 1426 & $(45,2)$ & 756 & $(53,0)$ \\
\hline Buenos & 1283 & $(40,6)$ & 716 & $(55,8)$ \\
\hline Incapacidad Social & 450 & $(14,2)$ & 294 & $(65,3)$ \\
\hline \multicolumn{5}{|c|}{ ESTADO FUNCIONAL (INDICE DE KATZ) } \\
\hline Independiente & 2445 & $(76,5)$ & 1345 & $(55,0)$ \\
\hline Dependencia moderada & 459 & $(14,4)$ & 268 & $(58,4)$ \\
\hline Dependencia severa & 293 & $(9,2)$ & 182 & $(62,1)$ \\
\hline \multicolumn{5}{|l|}{ MOVILIDAD } \\
\hline Plena & 2981 & $(93,0)$ & 1647 & $(55,2)$ \\
\hline Restringida & 223 & $(7,0)$ & 154 & $(69,1)$ \\
\hline \multicolumn{5}{|c|}{ DETERIORO COGNITIVO (MMSE) } \\
\hline No & 2329 & $(72,9)$ & 1210 & $(51,9)$ \\
\hline $\mathrm{Si}$ & 864 & $(27,1)$ & 587 & $(67,9)$ \\
\hline
\end{tabular}


Tabla 1 (continuación)

\begin{tabular}{|c|c|c|c|c|}
\hline & $n$ & $\%$ & Mujeres & (\%) \\
\hline \multicolumn{5}{|l|}{ DEPRESIÓN (GDS) } \\
\hline No & 2400 & $(81,1)$ & 1256 & $(52,3)$ \\
\hline Sí & 559 & $(18,9)$ & 401 & $(71,7)$ \\
\hline \multicolumn{5}{|l|}{ CALIDAD DE VIDA (QLI) } \\
\hline Buena & 2713 & $(85,9)$ & 1481 & $(54,6)$ \\
\hline Mala & 446 & $(14,1)$ & 294 & $(65,9)$ \\
\hline \multicolumn{5}{|l|}{ AUTOPERCEPCIÓN } \\
\hline \multicolumn{5}{|l|}{ DE SALUD } \\
\hline Muy Bien/Bien & 2652 & $(83,6)$ & 1416 & $(53,4)$ \\
\hline Mal/Muy Mal & 521 & $(16,4)$ & 367 & $(70,4)$ \\
\hline \multicolumn{5}{|l|}{ VISIÓN } \\
\hline Sin Déficit & 2532 & $(79,3)$ & 1383 & $(54,6)$ \\
\hline Déficit Leve & 486 & $(15,2)$ & 301 & $(61,9)$ \\
\hline Déficit Moderado/Severo & 176 & $(5,5)$ & 110 & $(62,5)$ \\
\hline \multicolumn{5}{|l|}{ AUDICIÓN } \\
\hline Sin Déficit & 2648 & $(82,8)$ & 1476 & $(55,7)$ \\
\hline Déficit Leve & 344 & $(10,7)$ & 185 & $(53,8)$ \\
\hline Déficit Moderado/Severo & 209 & $(6,5)$ & 138 & $(66,0)$ \\
\hline \multicolumn{5}{|l|}{ CAÍDAS } \\
\hline Ninguna & 2619 & $(82,3)$ & 1387 & $(53,0)$ \\
\hline Una o más & 565 & $(17,7)$ & 400 & $(70,7)$ \\
\hline \multicolumn{5}{|l|}{ INCONTINENCIA } \\
\hline No & 2679 & $(83,4)$ & 1442 & $(53,8)$ \\
\hline Sí & 509 & $(15,8)$ & 348 & $(68,4)$ \\
\hline \multicolumn{5}{|l|}{ VALORES PERDIDOS: } \\
\hline $\begin{array}{l}\text { Edad: } 8 \text {; Estado civil: } 17 \text {; } \\
\text { 10; Deterioro cognitivo: } 2 \\
\text { 30; Incontinencia: } 26 \text {. }\end{array}$ & $\begin{array}{l}\text { Escolar } \\
\text { oresión: }\end{array}$ & $\begin{array}{l}\text { omicilio: } \\
\text { de vida: }\end{array}$ & $\begin{array}{l}\text { sociales } \\
1 \text {; Visió }\end{array}$ & $\begin{array}{l}\text { 17; Movilidad } \\
\text { ón: 13; Caidas }\end{array}$ \\
\hline
\end{tabular}

betismo, incapacidad social, peor autopercepción de salud, dependencia funcional, deterioro cognitivo, depresión y mala calidad de vida.

Características de la hospitalización: Durante los 18 meses de seguimiento de la cohorte fueron hospitalizados en camas de agudos 410 individuos $(12,8 \%)$, que generaron 546 ingresos y 7.015 días de estancia. El
$75,3 \%$ de los individuos (309) ingresaron en una sola ocasión, el 17,8\% (73) dos veces, y el $6,8 \%$ (28) fueron hospitalizados en tres o más ocasiones.

En el Servicio de Geriatría (SG) ingresaron 168 pacientes $(30,7 \%)$, en los Servicios Médicos (SM) 204 (37,3\%) y en los Servicios Quirúrgicos (SQ) 174 (32,0\%). El promedio de edad fue significativamente mayor 
en geriatría (77,4 años) que en SM y SQ (73,6 y 73,7 años, respectivamente). Los ingresos programados fueron $112(20,5 \%)$, realizándose la mayoría de ellos en los SQ $(68,8 \%)$; la estancia media fue de 12,8 días (IC.95\%: 10,6-14,0), sin diferencias significativas entre servicios. Fallecieron un total de 44 pacientes $(8,1 \%)$, de los que 26 $(59,1 \%)$ estaban ingresados en el SG; la mortalidad en este servicio fue del $15,5 \%$ frente al 5,4\% en SM y al 4,0 en SQ.

Los diagnósticos más frecuentes (tabla 2) fueron los relacionados con el sistema cardio-circulatorio $(32,4 \%)$, respiratorio $(14,4 \%)$, digestivo $(13,8 \%)$. En los SQ predominaron los ingresos por accidentes, órganos de los sentidos, sistema músculoesquelético y génito-urinario, y en los SM y $\mathrm{SG}$ los ingresos por enfermedades cárdiovasculares, respiratorias, infecciosas y digestivas. Comparando los ingresos por cau- sas médicas entre geriatría y el resto de los $\mathrm{SM}$, en estos últimos predominaron los ingresos por enfermedades del aparato digestivo y neoplasias y en geriatría las enfermedades respiratorias y endocrinas.

En cuanto al estado de salud y las características personales de los pacientes hospitalizados (tabla 3), los SQ tuvieron una mayor proporción de pacientes más jóvenes, mujeres y con déficit leve de visión y audición que los no quirúrgicos, mientras que en estos últimos, (SM y $\mathrm{SG}$ ) existió una mayor frecuencia de pacientes con deterioro cognitivo, no existiendo diferencias en el resto de los indicadores estudiados. En el SG, respecto a los SM, se halló una significativa mayor proporción de pacientes mayores de 80 años, que vivían en residencias, no tenían pareja, con dependencia funcional moderada y severa, deterioro cognitivo, depresión, mala calidad de vida y escasos recursos sociales.

Tabla 2

Diagnósticos de alta por servicio $(\%)$

\begin{tabular}{|c|c|c|c|c|}
\hline & Geriatría & Médicos & Quirürgicos & Total \\
\hline INFECCIOSAS Y PARASITARIA & $7(58,3)$ & $5(41,7)$ & $0(0)$ & $12(2,3)$ \\
\hline NEOPLASIAS & $5(8,9)$ & $20(35,7)$ & $31(55,4)$ & $56(10,6)$ \\
\hline $\begin{array}{l}\text { ENDOCRINAS, NUTRICIONALES } \\
\text { Y METABÓLICAS }\end{array}$ & $7(70,0)$ & $2(20,0)$ & $1(10,0)$ & $10(1,9)$ \\
\hline HEMATOLÓGICAS & $2(33,3)$ & $3(50,0)$ & $1(16,7)$ & $6(1,1)$ \\
\hline TRASTORNOS MENTALES & $5(55,5)$ & $4(44,5)$ & $0(0)$ & $9(1,7)$ \\
\hline ORGANOS DE LOS SENTIDOS & $1(3,5)$ & $1(3,5)$ & $26(93,0)$ & $28(5,3)$ \\
\hline SISTEMA CARDIOCIRCULATORIO & $70(40,9)$ & $89(52,1)$ & $12(7,0)$ & $171(32,4)$ \\
\hline SISTEMA RESPIRATORIO & $52(68,4)$ & $24(31,6)$ & $0(0)$ & $76(14,4)$ \\
\hline SISTEMA DIGESTIVO & $7(9,6)$ & $33(45,2)$ & $33(45,2)$ & $73(13,8)$ \\
\hline SISTEMA GENITO-URINARIO & $3(15,0)$ & $5(25,0)$ & $12(60,0)$ & $20(3,8)$ \\
\hline PIEL Y TEJIDO SUBCUTÁNEO & $1(33,3)$ & $1(33,3)$ & $1(33,3)$ & $3(0,6)$ \\
\hline SISTEMA MÚSCULO ESQUELÉTICO & $1(6,7)$ & $3(20,0)$ & $11(73,3)$ & $15(2,8)$ \\
\hline $\begin{array}{l}\text { SÍNTOMAS Y SIGNOS Y } \\
\text { MAL DEFINIDOS }\end{array}$ & $3(25,0)$ & $6(50,0)$ & $3(25,0)$ & $12(2,3)$ \\
\hline $\begin{array}{l}\text { ACCIDENTES, LESIONES, } \\
\text { INTOXICACIONES }\end{array}$ & $1(2,7)$ & $5(13,5)$ & $31 \quad(83,8)$ & $37(7,0)$ \\
\hline
\end{tabular}


Tabla 3

Características demográficas y estado de salud de las personas ingresados por servicio

\begin{tabular}{|c|c|c|c|c|}
\hline & Geriatría & Médicos & Quirúrgico & Total \\
\hline \multicolumn{5}{|l|}{ SEXO* } \\
\hline Hombre & $86(30,9)$ & $117(42,1)$ & $75(27,0)$ & $278(50,9)$ \\
\hline Mujer & $82(30,6)$ & $87(32,5)$ & $99(36,9)$ & $268(49,1)$ \\
\hline \multicolumn{5}{|l|}{$\mathrm{EDAD}^{* *}$} \\
\hline $65-6$ & $19(16,5)$ & $58(50,4)$ & $38(33,0)$ & $115(21,4)$ \\
\hline $70-74$ & $41(28,3)$ & $57(39,3)$ & $47(32,4)$ & $145(27,0)$ \\
\hline $75-79$ & $29(25,4)$ & $44(38,6)$ & $41(36,0)$ & $114(21,2)$ \\
\hline$>80$ & $77(47,0)$ & $43(26,2)$ & $44(26,8)$ & $164(30,4)$ \\
\hline \multicolumn{5}{|l|}{ ESTADO CIVIL* } \\
\hline Casado & $90(25,6)$ & $147(41,8)$ & $115(32,7)$ & $352(54,7)$ \\
\hline Sin Pareja & $78(40,6)$ & $56(29,2)$ & $58(30,2)$ & $192(35,3)$ \\
\hline \multicolumn{5}{|l|}{ NIVEL DE ESTUDIOS } \\
\hline Analfabeto & $42(37,8)$ & $37(33,3$ & $32(28,8)$ & $111(20,5)$ \\
\hline Leer/Escribir & $113(31,1)$ & $135(37,2)$ & $115(31,7)$ & $363(67,1)$ \\
\hline Primarios/Superiores & $13(19,4)$ & $29(43,3)$ & $25(37,3)$ & $67(12,4)$ \\
\hline \multicolumn{5}{|l|}{ DOMICILIO** } \\
\hline Propio & $108(27,3)$ & $158(40,0)$ & $129(32,7)$ & $395(73,8)$ \\
\hline Cuidador & $32(32,3)$ & $37(37,4)$ & $30(30,3)$ & $99(18,5)$ \\
\hline Residencia & $25(61,0)$ & $5(12,2)$ & $11(26,8)$ & $41(7,7)$ \\
\hline \multicolumn{5}{|c|}{ RECURSOS SOCIALES* (OARS) } \\
\hline Excelentes & $59(28,5)$ & $90(43,5)$ & $58(28,0)$ & $207(39,0)$ \\
\hline Buenos & $61(27,7)$ & $83(37,7)$ & $76(34,5)$ & $220(41,4)$ \\
\hline Incapacidad Social & $41(39,4)$ & $29(27,9)$ & $34(32,7)$ & $104(19,6)$ \\
\hline \multicolumn{5}{|c|}{ ESTADO FUNCIONAL* (INDICE DE KATZ) } \\
\hline Independiente & $86(25,2)$ & $142(41,5)$ & $114(33,3)$ & $342(63,0)$ \\
\hline Dependencia Moderada & $38(38,8)$ & $32(32,7)$ & $28(28,6)$ & $98(18,0)$ \\
\hline Dependencia Severa & $43(41,7)$ & $29(28,2)$ & $31(30,1)$ & $103(19,0)$ \\
\hline \multicolumn{5}{|l|}{ MOVILIDAD } \\
\hline Plena & $143(30,1)$ & $184(38,7)$ & $148(31,2)$ & $475(87,2)$ \\
\hline Restringida & $25(35,7)$ & $20(28,6)$ & $25(35,7)$ & $70(12,8)$ \\
\hline \multicolumn{5}{|c|}{ DETERIORO COGNITIVO (MMSE)** } \\
\hline No & $75(21,5)$ & $147(42,1)$ & $127(36,4)$ & $349(64,4)$ \\
\hline Sí & $93(48,2)$ & $54(28,0)$ & $46(23,8)$ & $193(35,6)$ \\
\hline \multicolumn{5}{|l|}{ DEPRESIÓN (GDS)* } \\
\hline No & $84(24,9)$ & $136(40,4)$ & $117(34,7)$ & $337(71,5)$ \\
\hline Sí & $48(35,8)$ & $42(31,3)$ & $44(32,8$ & $134(28,5)$ \\
\hline
\end{tabular}


Tabla 3 (continuación)

\begin{tabular}{|c|c|c|c|c|}
\hline & Geriatría & Médicos & Quirúrgico & Total \\
\hline \multicolumn{5}{|l|}{ CALIDAD DE VIDA (QLI)** } \\
\hline Buena & $99(25,7)$ & $156(40,5)$ & $130(33,8)$ & $385(72,6)$ \\
\hline Mala & $64(44,1)$ & $40(27,6)$ & $41(28,3)$ & $145(27,4)$ \\
\hline \multicolumn{5}{|l|}{ AUTOPERCEPCIÓN } \\
\hline \multicolumn{5}{|l|}{ DE SALUD } \\
\hline Muy Bien/Bien & $124(31,1)$ & $144(36,1)$ & $131(32,8)$ & $399(74,7)$ \\
\hline Mal/Muy Mal & $37(27,4)$ & $56(41,5)$ & $42(31,1)$ & $135(25,3)$ \\
\hline \multicolumn{5}{|l|}{ VISIÓN* } \\
\hline Sin Déficit & $114(29,4)$ & $159(41,0)$ & $115(29,6)$ & $388(71,1)$ \\
\hline Déficit Leve & $30(29,7)$ & $24(23,8)$ & $47(46,5)$ & $101(18,5)$ \\
\hline Déficit Moderado/Severo & $24(42,1)$ & $21(36,8)$ & $12(21,1)$ & $57(10,4)$ \\
\hline \multicolumn{5}{|l|}{ AUDICIÓN* } \\
\hline Sin Déficit & $131(29,9)$ & $176(40,2)$ & $131(29,9)$ & $438(80,2)$ \\
\hline Déficit Leve & $21(33,3)$ & $12(19,0)$ & $30(47,6)$ & $63(11,5)$ \\
\hline Déficit Moderado/Severo & $16(35,6)$ & $16(35,6)$ & $13(28,9)$ & $45(8,2)$ \\
\hline \multicolumn{5}{|l|}{ CAÍDAS } \\
\hline Ninguna & $119(28,2)$ & $164(38,9)$ & $139(32,9)$ & $422(78,3)$ \\
\hline Una o más & $45(38,5)$ & $39(33,3)$ & $33(28,2)$ & $117(21,7)$ \\
\hline \multicolumn{5}{|l|}{ INCONTINENCIA } \\
\hline No & $128(29,4)$ & $165(37,9)$ & $142(32,6)$ & $435(79,7)$ \\
\hline Sí & $40(36,0)$ & $39(35,1)$ & $32(28,8)$ & $111(20,3)$ \\
\hline
\end{tabular}

${ }^{*} \mathrm{p}<0,05,{ }^{* *} p<0,001$. Valores perdidos: Edad: 8; Estado civil: 2; Nivel Escolarización: 5; Domicilio: 11; Recursos sociales: 15 ; I. Kazt: 3; Movilidad: 1; Deterioro Cognitivo: 4; Depresión: 75; QLI: 16; Salud: 12; Caídas: 7.

\section{DISCUSIÓN}

Los resultados de este estudio no muestran diferencias en estado de salud entre las personas mayores de 64 años ingresadas en servicios no quirúrgicos y quirúrgicos, pero los pacientes ingresados en geriatría respecto a los ingresados en SM o SQ tienen una edad media más elevada y peor estado de salud, resultados consistentes con el perfil genérico de los pacientes que ingresan en unidades geriátricas de agudos ${ }^{4,10,32}$. Previsiblemente relacionado con este peor estado de $\operatorname{salud}^{33,34}$, la mortalidad en geriatría fue 3 veces mayor que en el resto de los servicios. Sin embargo, contra lo que sería esperable a partir de este perfil ${ }^{33,35}$, la EM en geriatría no superó la de los otros servicios. A este respecto, los trabajos que han analizado la EM entre servicios médicos y geriátricos ofrecen resultados dispares ${ }^{36-38}$, probablemente en relación con las diferencias en la tipología de pacientes.

Desde una perspectiva local, los resultados de este estudin muestran, en primer lugar, que pese a la ausencia de protocolos explícitos, el servicio de geriatría estudiado ingresa pacientes de perfil geriátrico más acentuado que los SM y, en segundo lugar, que la asistencia en unidades específicas de geriatría no conlleva -pese al referido peor estado de salud de los pacientes- una duración de la estancia mas prolongada que la dc los SM. Otros aspectos de interés para valo- 
rar el impacto de este tipo de unidades, como los reingresos y estado de salud tras el alta $^{39}$ no fueron analizados en este estudio.

La mayor frecuencia de diagnósticos relacionados con el aparato circulatorio, respiratorio, digestivo y neoplasias coinciden con los encontrados en otros estudios ${ }^{2,40}$ para las personas de este grupo de edad. En cirugía los grupos diagnósticos más frecuentes son los que contienen procesos usualmente susceptibles de tratamiento quirúrgico: órganos de los sentidos (cataratas), accidentes y lesiones (fracturas), génito-urinario (patología genital en las mujeres y prostática en los hombres), neoplasias y digestivo (hernias). Las diferencias observadas en cuanto a los grupos diagnósticos entre SM y geriatría probablemente estén en relación con la organización y recursos disponibles en cada servicio (las arritmias ingresan en la unidad de cuidados intensivos, las neoplasias en la especialidad correspondiente, etc.), situación que orientaría a que en la decisión de ingreso en el servicio de geriatría u otro servicio médico, influye, además del estado de salud, el diagnóstico.

Respecto a las limitaciones de este estudio hay que señalar, en primer lugar, el bajo número de casos en algunos análisis -singularmente en el de ingresos por grupos diagnósticos- que no permite valorar aquellos grupos con menos personas. También hay que considerar los posibles sesgos por la perdida de los pacientes que ingresaron en hospitales de otras áreas, aunque dada la buena accesibilidad en el área de Toledo no se considera una limitación relevante. Respecto a la generalización de los resultados, hay que señalar que la existencia de un servicio de geriatría con 135 camas, que permite una asistencia diferenciada a los ancianos, no es frecuente en el entorno sanitario español, aspecto que conlleva dificultades para su generalización a otras áreas con menos recursos, que podrían tener un diferente perfil de pacientes si emplean criterios de selección más estrictos $^{39,41}$. Una segunda limitación pro- viene del hecho de que no están incluidas en el estudio las intervenciones realizadas con cirugía ambulatoria, muy desarrollada en el área sanitaria de Toledo, aspecto que podría suponer diferencias en el perfil de los pacientes quirúrgicos respecto a aquellos lugares sin este tipo de servicio. La tercera limitación está relacionada con la mortalidad ya que solamente se conoce la mortalidad hospitalaria y no se dispone de la mortalidad posterior al alta que, previsiblemente, incrementaría las cifras, dadas la edad elevada y el estado de salud de las personas de la muestra. Finalmente, aunque los instrumentos utilizados para la evaluación (Indice de Katz QLI, MMSE, GDS, y escala de recursos sociales de la OARS) muestran un buen poder discriminatorio para el ingreso en unidades de geriatría, hay que considerar los puntos de corte utilizados y la ponderación de la puntuación del MMSE según el nivel de educación utilizados en este trabajo, ya que en este ultimo caso, por ejemplo, la ponderación introducida disminuye ostensiblemente la proporción de la población con deterioro cognitivo.

Aunque son necesarios más estudios para identificar un perfil diferencial de las personas mayores de 64 años susceptibles de beneficiarse del ingreso en unidades específicas de Geriatría, los resultados de este estudio sugieren que las líneas de este pertil, junto a la mayor edad, incluyen una mayor proporción de problemas sociales (ancianos que viven solos, institucionalizados, escasos recursos sociales), menor independencia para realizar las actividades de la vida diaria, mas enfermedades mentales (deterioro del estado cognitivo y depresión) y menor puntuación en el QLI. Estos factores, más relacionados con el estado funcional y situación social que con los propios parámetros clínicos, van a requerir la utilización habitual de instrumentos de valoración, del tipo de los utilizados en este estudio, para mejorar las decisiones de admisión entre los servicios médicos y los servicios geriátricos. 


\section{BIBLIOGRAFÍA}

1. Instituto Nacional de Estadística. Proyecciones de la población española calculada a partir del Censo de Población de 1991. Madrid: INE; 1995.

2. Castellá X, Mompart A, Pérez G. La utilización de los hospitales de agudos por los ancianos. Cataluña, 1982-1990. Gac Sanit 1997; 11:259-265.

3. Hyattsville MD. Vital and Health Statistics: Health Data on Older Americans 1992. Department of Health and Human Services (US). Series III: Analytic and epidemiological studies $N^{\circ} 127$ 1993. DHHS Publication No 93-141.

4. Instituto Nacional de Salud. Criterios de ordenación de servicios para la atención sanitaria a las personas mayores. Madrid: Insalud;1995.

5. Guerrero M F, Alfonso J L, Sanchís B, Prado M J. ¿Utilización hospitalaria y ancianidad creciente en el usuario atendido?. Gac Sanit 1992;6:62-66.

6. Compañ L, Portella E. Impacto del envejecimiento de la población española en la utilización de servicios hospitalarios. Todo Hosp 1994;106:13-17.

7. Freeborn DK, Pope CR, Mullooly JP, McFarland BH. Consistently high users of medical care among the elderly. Med Care 1990;28:527-540.

8. Wolinsky FD, Johnson RJ. The use of health services by older adults. J Gerontol 1991; 46:S345-357.

9. Tomiak M, Berthelot JM, Mustard CA. A profile of health care utilization of the disabled population in Manitoba. Med Care 1998; 36:1383-1397.

10. González JI, Pérez del Molino J, Rodríguez L, Salgado A, Guillén F. Geriatría y asistencia geriátrica: para quién y cómo (I). Med Clin (Barc) 1991;96:183-188.

11. Winograd $\mathrm{CH}$, Gerety MB, Brown E, Kolodny $\mathrm{V}$. Targeting the hospitalized elderly for geriatric consultation. J Am Geriatr Soc 1988; 39:1113-1119.

12. Landefeld CS, Palmer RM, Kresevic DM, Fortinsky RH, Kowal J. A ramdomized trial of care in a hospital medical unit especially designed to improve the functional outcomes of acutely ill older patients. $\mathrm{N}$ Engl $\mathrm{J}$ Med 1995;332:1338-1344.

13. Stuck AE, Siu AL, Wieland GD, Adams J, Rubenstein LZ. Comprehensive geriatric assessment: a meta-analysis of controlled trials. Lancet $1993 ; 342: 1032-1036$.

14. Inouye SK, Acampora D, Miller RL, Fulmer T, Hurst LD, Cooney LM. The Yale Geriatric Carc Program: A model of care to prevent functional decline in hospitalized elderly patients. J Am Geriatr Soc 1993;41:1345-1352.

15. Covinsky KE, King IT, Quinn LM, Siddique R, Palmer R, Kresevic DM, Fortinsky RH, Kowal J, Landefeld CS. Do acute care for elders units increase hospital cost? A cost analysis using the hospital perspective. J Am Geriatr Soc 1997; $45: 729-734$.

16. Naughton BJ, Moran MB, Fcinglass J, Falconer J, Williams ME. Reducing hospital cost for the geriatric patient admitted from the emergency department: a randomized trial. J Am Geriatr Soc 1994;42: 1045-1049.

17. Teasdale TA, Schuman L, Snow E, Luchi RJ. A comparison of outcomes of geriatric cohorts receiving care in a geriatric assessment unit and on general medicine floors. J Am Geriatr Soc 1983;31:529-534.

18. Sager MA, Rudberg MA Jalaluddin M, Franke $\mathrm{T}$, Inouye $\mathrm{S}$, Landefeld $\mathrm{CS}$, Siebens $\mathrm{H}$, Winograd $\mathrm{CH}$. Hospital admission risk profile (HARP): identifying older patients at risk for functional decline following acute medical illness and hospitalization. J Am Geriatr Soc 1996;44:251-257.

19. Instituto Nacional de Salud. Memoria Anual del Insalud Toledo 1995. Toledo: Insalud; 1996.

20. Duke University Center for the Study of aging and Human development. Multifunctional assessment: the OARS methodology. Durham: Duke University; 1978.

21. Eiroa Patiño P, Vazquez-Vizoso FL, Veras Castro R. Discapacidades y necesidades de scrvicios en las personas mayores detectadas en la encuesta de salud OARS-Vigo. Med Clin (Barc) 1996;106:641-648.

22. Folstein MF, Folstcin S, McHugh PR. «MiniMental State». A practical method for grading the cognitive state of patients for the clinician. J Psychiatr Res 1975;12:189-198. 
23. García de Blas F, Gonzalez-Montalvo JI, Bermejo F, Colmenero C, Delgado I, Miguel I. Salud general, funcional y mental en la población anciana de tres barrios de Madrid. Rev Gerontol 1995; 5:81-89.

24. Tolosa E, Aloma J, Formable F. Criterios diagnósticos y escalas evacuativas en la enfermedad de Alzheimer. Rev Clin Esp 1987;181 (Supl 1):56-59.

25. Bermejo F, Díaz J, Olazarán J, Rivera J, Trincado R, Fernández C. Prevalencia de demencia en una muestra poblacional de ancianos en medio urbano. Análisis preliminar. Neurología 1995;10:418.

26. Pi J, Olivé JM, Esteban M. Mini Mental State Examination: asociación de la puntuación obtenida con la edad y grado de alfabetización en una población anciana. Med Clin (Barc) 1994;103:641-644.

27. Katz $S$, Ford $A B$, Moskowitz RW, Jackson BA, Jaffe MW, Cleveland MA. Studies of illness in the aged. The index of ADL: A standardized measure of biological and psychosocial function. J Am Med Assoc. 1963:185:914919.

28. Yesavage JA, Brink TL, Rose TL, Lum O, Huang V, Adey M, Leirer VO. Development and validation of a geriatric depression screening scale: a preliminary report. J Psychiatr Res 1982-83;17:37-49.

29. Sheikh JI, Yesavage JA. Geriatric Depression Scale (GDS): recent evidence and development of a shorter version. In: Clinical Gerontology. Binghamton: The Haworth Press; 1986: 165-173.

30. Spitzer WO, Dobson AJ, Hall J, Chesterman E, Levi J, Shepherd R, Battista RN, Catchlove $B R$. Measuring the quality of life of cancer patients: a concise QL-index for use by physicians. J Chronic Dis 1981; 34:585-597.

31. Carbonell MA. Adaptación y validación al español para mayores de 65 años del Indice de Calidad de Vida de Spitzer (QL INDEX). [tesis doctoral]. Universidad de Alcalá de Henares; 1998.
32. Gil Gregorio P. Unidad de agudos. En: Atención al anciano en el medio sanitario. Madrid: Editores Médicos SA, 1998:101-115.

33. Narain P, Rubenstein LZ, Wieland GD, Rosbrook B, Strome S, Pietruszka F, Morley JE. Predictors of immediate and 6-month outcomes in hospitalized elderly patients. The importance of functional status. J Am Geriatr Soc 1988;36:775-783.

34. Fried L, Kronmal R, Newman A, Bild D, Mittelmark M, Polak JF, Robbins JA, Gardin JM. Risk factors for 5-year mortality in older adults. The Cardiovascular Health Study. JAMA 1998;279:585-592.

35. Maguire PA, Taylor IC, Stout RW. Elderly patients in acute medical wards: factors predicting length of stay in hospital. BMJ 1986; 292:1251-1253.

36. Stewart DA, Burns JM, Beard K, Dall JL, Lennox IM, Roberts MA, Macfarlane GJ. The roles of general and geriatric medicine in the provision of acute medical care for elderly patients. Health Bull (Edinb) 1992;50:259-66.

37 .-Pawlson LG. Hospital length of stay of frail elderly patients. Primary care by general internists versus geriatricians. J Am Geriatr Soc 1988;39:202-208.

38. Flamer HE, Christophidis N, Margetts C, Ugoni A, McLean AJ. Extended hospital stays with increasing age: the impact of an acute geriatric unit. Med J Aust 1996;164:10-13.

39. González Montalvo JI. Alternativas a la hospitalización convencional en el anciano. En: Gestión Sanitaria Innovaciones y desafíos. Barcelona: Ed: Masson SA;1998.p.372-394

40. Ministerio de Sanidad y Consumo. Sistema Nacional de Salud año 1996. Estadísticas de referencia estatal de los sistemas de agrupación de registros de pacientes (GDR). Madrid: Ministerio de Sanidad y Consumo;1997.

41. Sager MA, Franke T, Inouye SK, Landefeld S, Morgan T, Rudberg MA, Siebens H, Winograd $\mathrm{CH}$. Functional outcomes of acute medical illness and hospitalization in older persons. Arch Intern Med 1996;156: 645-652. 